\title{
Mortality After Invasive Second Breast Cancers Following Prior Radiotherapy for DCIS
}

\author{
Puyao C. Li, MD ${ }^{a, b, c}$; Zilu Zhang, MS ${ }^{b}$; Angel M. Cronin, MS'; and Rinaa S. Punglia, MD, MPHa,b
}

\begin{abstract}
Background: Women with a history of ductal carcinoma in situ (DCIS) are at increased risk for developing a second breast cancer (SBC). A prior meta-analysis of randomized studies of radiotherapy (RT) for DCIS has shown a trend toward increased breast cancer-specific mortality after SBC, but it did not have the power needed to detect a significant difference, due to a limited number of recurrences. This study sought to evaluate the impact of RT for DCIS on mortality after SBC in a larger cohort. Patients and Methods: Using the SEER database, 3,407 patients were identified who received breastconserving therapy with or without RT for primary DCIS in 2000 through 2013 and subsequently developed a stage I-III invasive SBC within the same time period. Fine-Gray competing risk models were used to study the association between receipt of RT and mortality after SBC. Results: Prior RT was found to be associated with higher rates of breast cancer-specific mortality (hazard ratio [HR], 1.70; $95 \% \mathrm{Cl}, 1.18-2.45 ; P=.005)$, even after controlling for cancer stage. Interaction analysis suggested that this risk trended higher in patients with ipsilateral versus contralateral $\mathrm{SBC}(\mathrm{HR}, 2.07$ vs $1.26 ; P=16)$. Furthermore, compared with patients who developed contralateral SBC, those with ipsilateral SBC were younger $(P<.001)$ and more often lacked estrogen receptor expression $(P<.001)$. Conclusions: Patients who previously received RT for DCIS had higher mortality after developing an invasive SBC than those who did not receive RT. This finding may have implications for initial treatment decisions in the management of DCIS.
\end{abstract}

J Natl Compr Canc Netw 2019;17(11):1367-1371 doi: $10.6004 /$ jnccn.2019.7323

\footnotetext{
a'Brigham and Women's Hospital, 'bana-Farber Cancer Institute, and
} 'Massachusetts General Hospital, Boston, Massachusetts.

\section{Background}

Women with a history of ductal carcinoma in situ (DCIS) are at increased risk for developing a second breast cancer (SBC) in either the ipsilateral or contralateral breast. ${ }^{1,2}$ Many women receive breast-conserving surgery (BCS) with adjuvant radiotherapy (RT), as recommended in the NCCN Guidelines. ${ }^{3}$ Adjuvant RT in this setting has been shown to decrease the risk of local recurrence, including development of an invasive SBC, but not mortality. ${ }^{4-7}$ However, breast cancer-specific survival is compromised among women who hvae an ipsilateral invasive cancer diagnosis after DCIS. ${ }^{8}$ The Early Breast Cancer Trialists' Collaborative Group (EBCTCG) meta-analysis of 3,729 women with DCIS enrolled in 4 randomized trials included 92 women who developed invasive SBC in the ipsilateral breast. Despite fewer incidences of SBC after RT in the ipsilateral breast, a trend toward higher breast cancerspecific mortality after RT was reported, but power to detect a difference was limited by the relatively small number of patients with SBC. ${ }^{4}$ Using a larger cohort in SEER, we sought to further evaluate the relationship between prior RT and mortality. In comparing ipsilateral and contralateral SBCs, we also sought to better understand the impact of RT on the characteristics and prognosis of SBC.

\section{Patients and Methods}

Using the SEER database, 3,407 patients were identified who received BCS \pm RT for primary DCIS in 2000 through 2013 and subsequently developed a stage I-III invasive SBC within the same time period (Figure 1). Patients aged $<40$ years who did not receive BCS, had unknown RT status, had bilateral or unknown laterality of primary or second breast cancer, and/or had stage IV SBC after DCIS were excluded. Differences in patient characteristics by laterality of SBC were assessed using chi-square and Wilcoxon rank sum tests. We assessed the association between time to breast cancer-specific death and receipt of RT for primary DCIS using Fine-Gray competing risk models, ${ }^{9}$ and adjusted for prespecified covariates of age at SBC diagnosis, interval between 


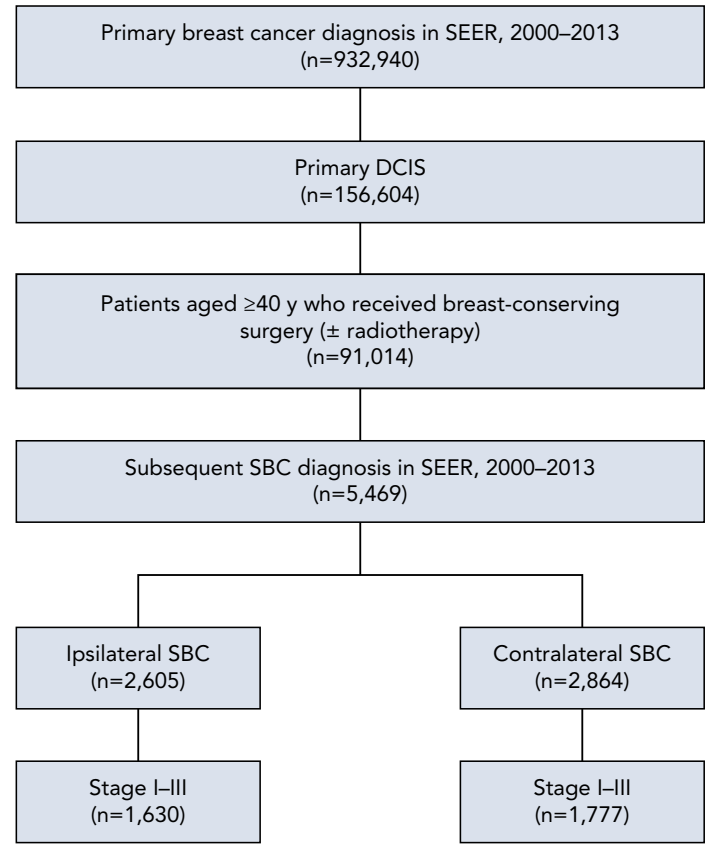

Figure 1. Flow diagram illustrating study population selection. Abbreviations: DCIS, ductal carcinoma in situ; SBC, second breast cancer.

primary and second breast cancer, and characteristics of the SBC (laterality, estrogen receptor [ER] status, stage, and year of diagnosis). Death by other causes was considered the competing risk event. Survival length was measured as the time after SBC diagnosis until death or December 31, 2013, whichever came first. A model with the interaction term was generated to further explore whether laterality of the SBC modified the effect of RT on mortality. Analyses were performed using STATA, version 15 (StataCorp LLP).

\section{Results}

A total of 150 deaths due to breast cancer were identified in the cohort: 61 among patients receiving BCS alone and 89 among those receiving BCS + RT for primary DCIS. Another 204 deaths were attributed to other causes (85 among patients receiving BCS alone and 119 among patients receiving BCS + RT for primary DCIS). Median follow-up for survivors from time of SBC diagnosis was 43 months (range, 1-158 months).

Patient characteristics, compared based on treatment of primary DCIS, are listed in Table 1. Patients who received BCS + RT for DCIS had fewer ipsilateral SBC diagnoses but more contralateral SBC diagnoses compared with those who received BCS alone $(P<.001)$. The interval from primary breast cancer to SBC was slightly longer for patients who received RT $(P=.02)$.

\begin{tabular}{|c|c|c|c|}
\hline & $\begin{array}{l}\text { BCS Alone } \\
(\%)\end{array}$ & $\begin{array}{c}\text { BCS + RT } \\
(\%)\end{array}$ & $P$ Value ${ }^{a}$ \\
\hline Total, $\mathrm{n}$ & 1,490 & 1,917 & \\
\hline Laterality of SBC & & & $<.001$ \\
\hline Ipsilateral & 58 & 40 & \\
\hline Contralateral & 42 & 60 & \\
\hline Age at SBC, y & & & $<.001$ \\
\hline $40-49$ & 10 & 10 & \\
\hline $50-59$ & 24 & 30 & \\
\hline $60-69$ & 27 & 31 & \\
\hline $70-79$ & 28 & 23 & \\
\hline$\geq 80$ & 11 & 6 & \\
\hline ER status of SBC & & & $<.001$ \\
\hline Positive & 6 & 5 & \\
\hline Negative & 80 & 76 & \\
\hline Unknown & 14 & 19 & \\
\hline Stage of SBC & & & .51 \\
\hline I & 66 & 68 & \\
\hline II & 27 & 25 & \\
\hline III & 7 & 7 & \\
\hline $\begin{array}{l}\text { Interval from primary to } \\
\text { SBC, median (range), y }\end{array}$ & $4.3(0.5-13.8)$ & $4.7(0.5-13.6)$ & .02 \\
\hline
\end{tabular}

Abbreviations: $\mathrm{BCS}$, breast-conserving surgery; DCIS, ductal carcinoma in situ; $E R$, estrogen receptor; RT, radiotherapy; SBC, second breast cancer.

a $P$ values were determined by chi-square and Wilcoxon rank sum tests.

Patients who received BCS + RT were slightly younger $(P<.001)$ with different ER status $(P<.001)$ than those who received BCS alone.

Patient characteristics by laterality of SBC were also examined. Compared with patients who developed contralateral SBC, patients who developed ipsilateral SBC were younger at the time of their primary and secondary diagnoses $(P<.001)$, were less likely to have ER expression for both diagnoses $(P<.001)$, and had longer interval to SBC $(P<.001)$.

The 5-year cumulative incidence of breast cancerspecific death after development of SBC was higher with prior RT than with no prior RT: $8.0 \%$ versus $4.7 \%$ for ipsilateral SBC (Figure 2A) and 5.3\% versus $4.2 \%$ for contralateral SBC (Figure 2B). In a multivariable competing risk analysis adjusting for age, ER status, and disease stage, prior use of RT in the treatment of primary breast cancer remained significantly associated with increased cancer-specific mortality (hazard ratio $[\mathrm{HR}], 1.70 ; 95 \% \mathrm{CI}, 1.18-2.45 ; P=.005)$. Interaction analysis suggested that this association differed by laterality of SBC (HR, 2.07 for ipsilateral vs $1.26[2.07 \times$ $0.61=1.26]$ for contralateral), although the interaction 
A

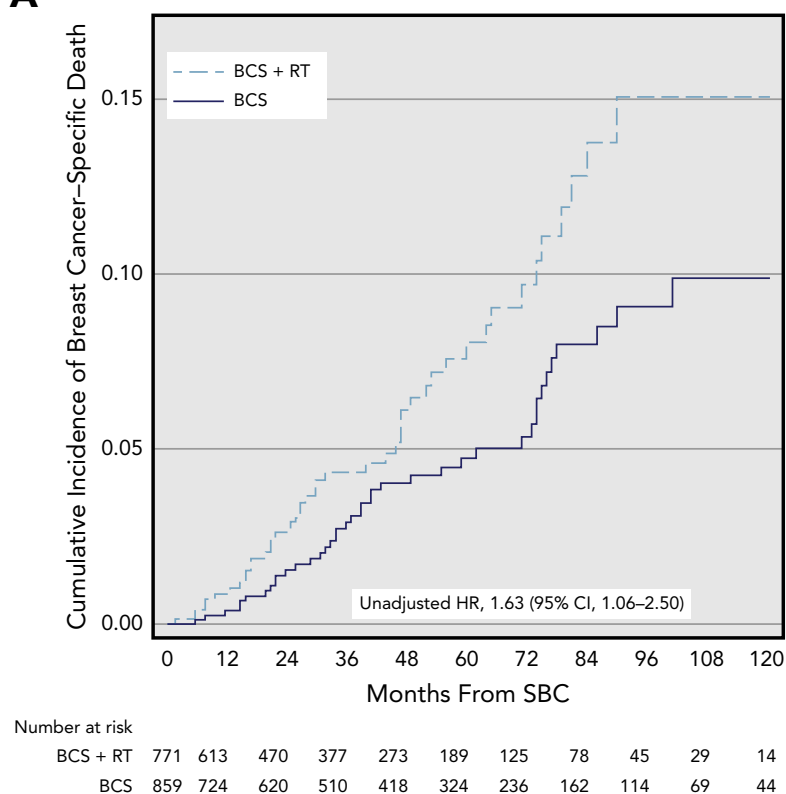

B

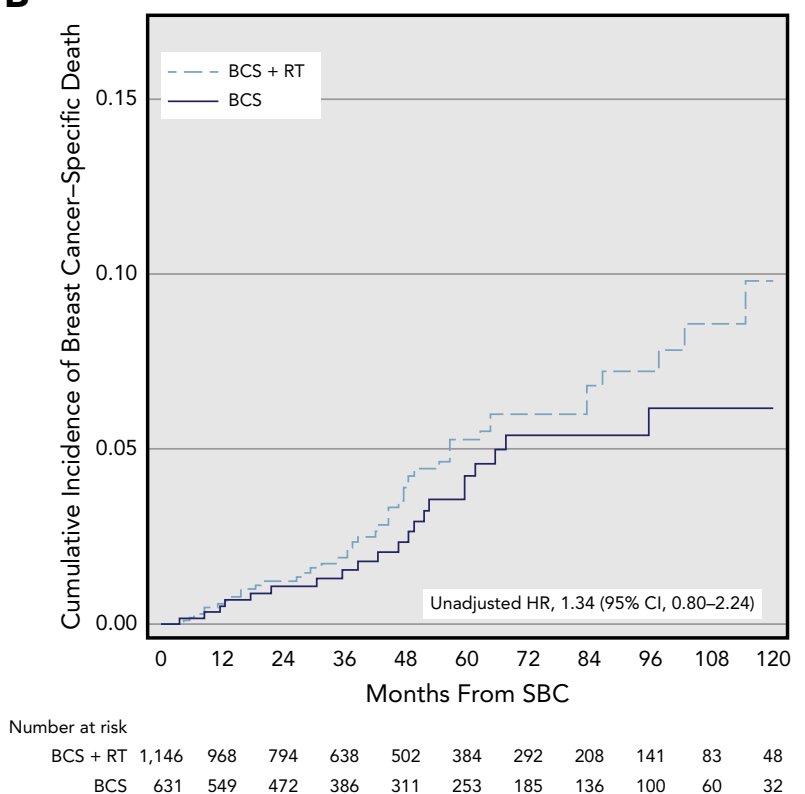

Figure 2. Cumulative incidence of breast cancer-specific death after an (A) ipsilateral or (B) contralateral stage I-III SBC in patients receiving BCS for primary ductal carcinoma in situ. Death of other causes is a competing risk event.

Abbreviations: BCS, breast-conserving surgery; HR, hazard ratio; RT, radiotherapy; SBC, second breast cancer.

did not reach statistical significance $(P=.16)$. Factors independently associated with breast cancer-specific mortality after SBC included age at SBC diagnosis $(P<.001)$ and ER status $(P<.001)$ and stage of SBC $(P<.001)$ (Table 2$)$.

\section{Discussion}

Among patients with invasive SBC after a DCIS diagnosis, prior RT was associated with increased breast cancerspecific mortality, with an HR of 2.07 for ipsilateral SBC even after controlling for disease stage and other factors known to impact survival. This finding corroborates the trend reported in the EBCTCG meta-analysis of randomized trials, which also found increased mortality after ipsilateral SBC in women who received RT initially for DCIS despite fewer invasive recurrences in the RT arm (92 women who received RT vs 204 who did not). ${ }^{4}$ The power to detect a statistically significant difference was limited by the small number of patients who developed invasive SBC in the meta-analysis, whereas in our study, the larger numbers of patients available in the SEER dataset provided much greater power to analyze this difference.

One factor likely contributing to the finding of increased mortality associated with ipsilateral SBC after prior RT is that prior radiation to the ipsilateral breast limits subsequent salvage options in the same breast. In our cohort, the proportion of patients receiving RT for contralateral SBC was higher than the proportion receiving RT for ipsilateral SBC after DCIS $(P<.001)$. Another hypothesis that may explain this finding is that BCS followed by RT is less effective in preventing recurrence of invasive disease that is more aggressive biologically, and therefore prior treatment with RT may in fact confer a worse prognosis among patients who develop an invasive SBC. Indeed, our findings showed that patients who developed ipsilateral SBC were younger at the time of SBC development and had breast cancers that were more likely to lack ER expression, characteristics that are associated with more aggressive cancer biology. These possible explanations for the increased mortality associated with prior RT are not mutually exclusive, and both may be true.

Regardless of the underlying reason, higher mortality associated with ipsilateral SBC after prior RT is a concerning finding that merits further investigation. This outcome may influence clinical decision-making regarding initial therapy for DCIS and highlights the importance of a nuanced discussion with each patient before treatment, taking into account individual patient characteristics and preferences. We believe that practice guidelines should encourage this discussion. Indeed, a previous study examining decision-making regarding use of RT for DCIS revealed that the optimal treatment strategy depends on an individual patient's utility or preference for specific health states. ${ }^{10}$ Furthermore, our 


\begin{tabular}{|c|c|c|c|c|}
\hline & \multicolumn{4}{|c|}{ Association Between Covariates and Breast Cancer-Specific Death ${ }^{a}$} \\
\hline & \multicolumn{2}{|c|}{ Model Without Interaction } & \multicolumn{2}{|c|}{ Model With Interaction } \\
\hline & HR $(95 \% \mathrm{Cl})$ & $P$ Value & HR $(95 \% \mathrm{Cl})$ & $P$ Value \\
\hline \multicolumn{5}{|l|}{ Treatment of primary DCIS } \\
\hline BCS alone & Ref & & Ref & \\
\hline $\mathrm{BCS}+\mathrm{RT}$ & $1.70(1.18-2.45)$ & .005 & $2.07(1.32-3.26)$ & .002 \\
\hline \multicolumn{5}{|l|}{ Laterality of SBC } \\
\hline Ipsilateral & Ref & & Ref & \\
\hline Contralateral & $0.60(0.42-0.86)$ & .005 & $0.82(0.47-1.42)$ & .48 \\
\hline Interaction: RT and laterality of SBC & - & - & $0.61(0.31-1.21)$ & .16 \\
\hline Age at $\mathrm{SBC}, \mathrm{y}$ & & $<.001$ & & $<.001$ \\
\hline $40-49$ & Ref & & Ref & \\
\hline $50-59$ & $1.05(0.56-1.97)$ & & $1.05(0.56-1.98)$ & \\
\hline $60-69$ & $1.68(0.92-3.07)$ & & $1.68(0.92-3.07)$ & \\
\hline $70-79$ & $1.81(0.97-3.37)$ & & $1.81(0.97-3.37)$ & \\
\hline$\geq 80$ & $3.51(1.74-7.09)$ & & $3.55(1.77-7.14)$ & \\
\hline ER status of SBC & & $<.001$ & & $<.001$ \\
\hline Positive & Ref & & Ref & \\
\hline Negative & $2.13(1.47-3.09)$ & & $2.13(1.47-3.09)$ & \\
\hline Unknown & $1.96(1.14-3.35)$ & & $1.98(1.15-3.40)$ & \\
\hline Stage of SBC & & $<.001$ & & $<.001$ \\
\hline I & Ref & & Ref & \\
\hline II & $3.08(2.08-4.57)$ & & $3.11(2.10-4.60)$ & \\
\hline III & $10.27(6.89-15.3)$ & & $10.52(7.04-15.7)$ & \\
\hline Interval from primary to $S B C$ (HR per $y)$ & $0.97(0.90-1.05)$ & .51 & $0.97(0.90-1.05)$ & .47 \\
\hline
\end{tabular}

Abbreviations: $B C S$, breast-conserving surgery; DCIS, ductal carcinoma in situ; ER, estrogen receptor; HR, hazard ratio; RT, radiotherapy; SBC, second breast cancer aHRs were calculated with Fine-Gray competing risk models. Models were adjusted for all variables shown in the table.

results suggest that patients who develop SBC after prior RT may benefit from intensified or alternative treatment as a strategy to improve survival.

Limitations of this study include its retrospective nature and those inherently associated with SEER database analyses. There are multiple unmeasured potential confounders, such as surgical margin status, endocrine therapy, patient comorbidities, reasons for treatment selection, and salvage therapy, not reported in the SEER database that may have influenced overall results. ${ }^{11,12}$ Furthermore, selection bias in the use of RT is likely. However, examining outcomes after SBC rather than after initial treatment and comparing ipsilateral and contralateral SBCs helped reduce the impact of this bias. SEER is thought to underreport receipt of RT, ${ }^{11,12}$ but any misclassification or underascertainment of its use would be expected to underestimate rather than overstate its effect. Nevertheless, we urge cautious interpretation of the clinical implications of our findings.

\section{Conclusions}

This study provides evidence that patients who previously received RT for DCIS had higher mortality after developing an invasive SBC than those who did not receive RT. This increase was particularly pronounced in patients with ipsilateral versus contralateral SBC. These findings may have implications for treatment decision-making in DCIS and after development of SBC, and highlight the value of a careful discussion with patients before treatment.

Submitted February 26, 2019; accepted for publication May 28, 2019.

Author contributions: Study concept: Li, Cronin, Punglia. Formal analysis: All authors. Supervision: Punglia. Methodology: Cronin, Punglia. Manuscript preparation, original: Li, Cronin. Manuscript preparation, revision: Zhang. Critical revision: All authors.

Disclosures: The authors have not received any financial consideration from any person or organization to support the preparation, analysis, results, or discussion of this article.

Correspondence: Rinaa S. Punglia, MD, MPH, Dana-Farber Cancer Institute, Center for Outcomes and Policy Research, 450 Brookline Avenue, Boston, MA 02115. Email: rpunglia@partners.org 


\section{References}

1. Bijker N, Meijnen $\mathrm{P}$, Peterse $\mathrm{JL}$, et al. Breast-conserving treatment with or without radiotherapy in ductal carcinoma-in-situ: ten-year results of European Organisation for Research and Treatment of Cancer randomized phase III trial 10853 - a study by the EORTC Breast Cancer Cooperative Group and EORTC Radiotherapy Group. J Clin Oncol 2006;24:3381-3387.

2. Houghton J, George WD, Cuzick J, et al. Radiotherapy and tamoxifen in women with completely excised ductal carcinoma in situ of the breast in the UK, Australia, and New Zealand: randomised controlled trial. Lancet 2003;362:95-102

3. Gradishar WJ, Anderson BO, Abraham J, et al. NCCN Clinical Practice Guidelines in Oncology: Breast Cancer. Version 4.2018. To view the most recent version visit NCCN.org. Accessed February 22, 2019.

4. Early Breast Cancer Trialists' Collaborative Group (EBCTCG); Correa C, McGale $\mathrm{P}$, et al. Overview of the randomized trials of radiotherapy in ductal carcinoma in situ of the breast. J Natl Cancer Inst Monogr 2010;2010:162-177.

5. Darby S, McGale P, Correa $\mathrm{C}$, et al. Effect of radiotherapy after breastconserving surgery on 10-year recurrence and 15-year breast cancer death: meta-analysis of individual patient data for 10,801 women in 17 randomised trials. Lancet 2011;378:1707-1716.

6. Narod SA, lqbal J, Giannakeas V, et al. Breast cancer mortality after a diagnosis of ductal carcinoma in situ. JAMA Oncol 2015;1:888-896.
7. Sagara Y, Freedman RA, Vaz-Luis I, et al. Patient prognostic score and associations with survival improvement offered by radiotherapy after breast-conserving surgery for ductal carcinoma in situ: a population-based longitudinal cohort study. J Clin Oncol 2016;34: 1190-1196.

8. Wapnir IL, Dignam JJ, Fisher B, et al. Long-term outcomes of invasive ipsilateral breast tumor recurrences after lumpectomy in NSABP B-17 and B-24 randomized clinical trials for DCIS. J Natl Cancer Inst 2011;103: 478-488.

9. Fine JP, Gray RJ. A proportional hazards model for the subdistribution of a competing risk. J Am Stat Assoc 1999;94:496-509.

10. Raldow $A C$, Sher $D$, Chen $A B$, et al. Cost effectiveness of the Oncotype DX DCIS score for guiding treatment of patients with ductal carcinoma in situ. J Clin Oncol 2016;34:3963-3968.

11. Walker GV, Grant SR, Guadagnolo BA, et al. Disparities in stage at diagnosis, treatment, and survival in nonelderly adult patients with cancer according to insurance status. J Clin Oncol 2014;32: 3118-3125

12. Jagsi R, Abrahamse $P$, Hawley $S T$, et al. Underascertainment of radiotherapy receipt in Surveillance, Epidemiology, and End Results registry data. Cancer 2012;118:333-341. 